OPEN ACCESS

Edited by:

Zeng-Yu Wang,

Qingdao Agricultural University, China

Reviewed by:

Masami Yokota Hirai,

RIKEN Center for Sustainable

Resource Science (CSRS), Japan

Jim Tokuhisa,

Virginia Tech, United States

*Correspondence:

Henning Frerigmann

henning.frerigmann@uni-koeln.de orcid.org/0000-0002-7067-2721

Tamara Gigolashvill

t.gigolashvili@uni-koeln.de ag-gigolashvili.botanik.uni-koeln.de orcid.org/0000-0002-0416-4796

Specialty section

This article was submitted to Plant Metabolism and Chemodiversity, a section of the journal Frontiers in Plant Science

Received: 13 March 2021 Accepted: 27 May 2021 Published: 01 July 2021

Citation:

Frerigmann $\mathrm{H}$, Hoecker $\mathrm{U}$ and Gigolashvili T (2021) New Insights on the Regulation of Glucosinolate Biosynthesis via COP1 and DELLA

Proteins in Arabidopsis Thaliana.

Front. Plant Sci. 12:680255

doi: 10.3389/fpls.2021.680255

\section{New Insights on the Regulation of Glucosinolate Biosynthesis via COP1 and DELLA Proteins in Arabidopsis Thaliana}

\author{
Henning Frerigmann ${ }^{1 *}$, Ute Hoecker ${ }^{2}$ and Tamara Gigolashvili ${ }^{2 *}$ \\ ${ }^{1}$ Department of Plant-Microbe Interactions and Cluster of Excellence on Plant Sciences, Max Planck Institute for Plant \\ Breeding Research, Cologne, Germany, ${ }^{2}$ BioCenter, Botanical Institute and Cluster of Excellence on Plant Sciences, \\ University of Cologne, Cologne, Germany
}

The biosynthesis of defensive secondary metabolites, such as glucosinolates (GSLs), is a costly process, which requires nutrients, ATP, and reduction equivalents, and, therefore, needs well-orchestrated machinery while coordinating defense and growth. We discovered that the key repressor of light signaling, the CONSTITUTIVE PHOTOMORPHOGENIC 1/SUPPRESSOR OF PHYTOCHROME A-105 (COP1/SPA) complex, is a crucial component of GSL biosynthesis regulation. Various mutants in this COP1/SPA complex exhibited a strongly reduced level of GSL and a low expression of jasmonate (JA)-dependent genes. Furthermore, cop1, which is known to accumulate DELLA proteins in the dark, shows reduced gibberellin (GA) and JA signaling, thereby phenocopying other DELLA-accumulating mutants. This phenotype can be complemented by a dominant gain-of-function allele of MYC3 and by crossing with a mutant having low DELLA protein levels. Hence, SPA1 interacts with DELLA proteins in a yeast two-hybrid screen, whereas high levels of DELLA inhibit MYC function and suppress JA signaling. DELLA accumulation leads to reduced synthesis of GSL and inhibited growth. Thus, the COP1/SPA-mediated degradation of DELLA not only affects growth but also regulates the biosynthesis of GSLs.

Keywords: COP1/SPA, DELLA, gibberellin, glucosinolate, jasmonate, MYC2, MYC3, MYC4

The loss of the COP1/SPA complex affects signaling downstream of DELLAs, attenuates jasmonate response, decreases MYC activity, and reduces glucosinolate levels in Arabidopsis.

\section{INTRODUCTION}

Recent years have seen the reintroduction of ecological theories, focusing on the interplay between growth and immunity, into studies on molecular plant biology (Züst et al., 2011; Yang et al., 2012; Campos et al., 2016; Kliebenstein, 2016; Major et al., 2020). While the knowledge on the regulation of defense metabolites is further expanded, the coordination of synthesis of these compounds with other needs of plants still requires more attention. Jasmonate (JA) coordinates immune and growth responses to increase plant survival upon changing environmental cues. Research on the effects of phyB inactivation on JA signaling suggests that the effects of JA on growth and defense can use partially divergent signaling elements and, therefore, are not directly linked in a cause-effect 
relationship (Howe et al., 2018; Ballaré and Austin, 2019). Light is not only a source of energy but is also an important signal for resource allocation (Huot et al., 2014). As such, the presence of other plants competing for light [sensed by a low red (R) to far-red (FR) light ratio] triggers rapid elongation growth and consumes metabolic resources that could otherwise be invested in the production of defense compounds. Plants have evolved adaptation mechanisms for mediating the balance in the "dilemma to grow or defend." The phytohormones JA and gibberellin (GA) are fundamentally important in facilitating the rapid adjustment of plant responses to the changing environment (Hou et al., 2013).

Recent studies suggest that the hormone-linked transcriptional network is hardwired to attenuate growth upon activation of JA signaling, and thus, the growth-defense antagonism is not caused by constraints on the availability of metabolic resources that fuel growth and defensive processes (Campos et al., 2016). This was revealed by a suppressor screen of the jazQ (jazQ being defective in JAZ1, JAZ3, JAZ4, JAZ9, and JAZ10) Arabidopsis mutant, in which the growth-defense trade-off was uncoupled. The jazQ mutant exhibits constitutive growth-defense antagonism (reduced growth with enhanced defense). The jazQ phyB mutant is morphologically larger than $j a z Q$ and Col-0 but retains the high level of insect resistance of jazQ (Campos et al., 2016).

While Campos et al. (2016) provide evidence that PIF and MYC transcription factors (TFs) are simultaneously activated in $p h y B j a z Q$, the role of DELLA in this process is still highly debated and is worth having a closer look at. Arabidopsis genome encodes five DELLA proteins with distinct but redundant functions: GAI, RGA, RGL1, RGL2, and RGL3. Contrary to the ability of the $p h y B$ mutations to completely uncouple the mild growth-defense phenotypes in $j a z Q$, phyB null alleles only weakly alleviated the growth and reproductive defects in the jazD (jaz1jaz7, jaz9, jaz10, and jaz13) mutants (Major et al., 2020). The molecular mechanism of JA-induced biomass reduction therefore continues to be intensely studied. Some recent literature studies (Major et al., 2020; Ortigosa et al., 2020) suggested that MYC2 targeting other genes can be involved in this process.

An existing model highlighting the interplay between JA, phyB, and GA (presented in Figure 1B) shows that phyB can perceive changes in the ratio of R:FR light and integrate the shade-avoidance response into GA-JA crosstalk. GA stimulates cell extension growth by promoting the degradation of DELLA proteins that repress PHYTOCHROMEINTERACTING FACTOR (PIF) TFs (Feng et al., 2008). Analogously, JA triggers defense responses via the COI1mediated depletion of JAZ repressors, which interfere with the function of MYC TFs (Fernández-Calvo et al., 2011). In the absence of JA, JAZ repressors bind to MYCs and inhibit the interaction between the MYCs and their targets, attenuating the potential of MYCs to activate the glucosinolate (GSL) pathway genes. JAZ proteins interact directly with DELLA repressors of GA signaling (Hou et al., 2010; Yang et al., 2012); thereby, JA-induced JAZ degradation can modulate the growth-defense balance by increasing the repressive activity of DELLA proteins on growth-promoting TFs. The interaction between JAZ and
DELLA proteins prevent these repressors from inhibiting their cognate TFs and enable a reciprocal antagonism between the JA and GA pathways (Hou et al., 2010; Wild et al., 2012).

However, upon light perception, phyB and other photoreceptors inactivate another class of repressors in addition to PIF proteins, which suppress light signaling in dark-grown and shaded Arabidopsis: the CONSTITUTIVE PHOTOMORPHOGENIC 1/SUPPRESSOR OF PHYTOCHROME A-105 (COP1/SPA) complex(es) (Hoecker, 2017). In Arabidopsis, COP1 is a single-copy gene, whereas SPA proteins are encoded by a small gene family of four genes (SPA1-SPA4) with overlapping, but partially distinct, functions. The cop1 and multiple spa mutants exhibit constitutive light signaling and show features of light-grown seedlings in complete darkness. The COP1/SPA complex is an E3 ubiquitin ligase that targets positive regulators of light signaling, mainly TFs, for degradation in darkness. Since phyB is an upstream regulator of COP1/SPA and appears to be involved in balancing the JA-GA phytohormone network, we questioned whether the COP1/SPA complex might also be a contributory component. For example, the shade-induced increase in GA level is thought to be regulated via shade-induced phyB inactivation [Figure 1B; reviewed by Casal (2013)]. However, the pea ortholog of COP1 is also required to sustain high levels of GA in dark-grown seedlings, probably via the ubiquitination of an ELONGATED HYPOCOTYL 5 (HY5) ortholog (Weller et al., 2009). Interestingly, recent findings (Ortigosa et al., 2020) have additionally demonstrated that MYC2, regulating GSLs response, also directly targets the central regulator of photomorphogenesis HY5. In this study, we, therefore, investigated the potential involvement of the COP1/SPA complex in the intricate hormone network that regulates GSL biosynthesis.

Glucosinolates are among the best-studied defense compounds in Arabidopsis, which are also attributed to phytoanticipins and phytoalexins (Piasecka et al., 2015). Despite their constitutive synthesis, plants integrate abiotic and biotic environmental factors with internal signals to increase specific GSL-mediated defense (Burow, 2016). In particular, the relative spatial abundance of indolic GSLs (IGs) and their MYB regulators (MYB34, MYB51, and MYB122) reflects changes in their response to different stimuli, such as phytohormones (Frerigmann and Gigolashvili, 2014a) or sulfur limitation (Frerigmann and Gigolashvili, 2014b). Most GSL biosynthesis genes have been identified; thus, the regulation of these compounds serves as a model system for secondary metabolites (Burow, 2016; Frerigmann, 2016). The entire GSL biosynthesis pathway is transcriptionally regulated by a group of six homologous MYB TFs and MYC proteins (Frerigmann, 2016; Supplementary Figure 1). These MYC TFs, of which MYC2 is the most prominent member, are also key regulators of JA signaling. Thus, the $m y c 2 / 3 / 4$ mutant lacks both the transcription of JA-induced genes, such as JAZ10 and VSP2 (Fernández-Calvo et al., 2011) (Figure 1A), as well as the expression of genes involved in the synthesis of IG (such as CYP79B3 and CYP83B1) and aliphatic GSLs (AG) (such as CYP83A1) (Frerigmann et al., 2014). However, MYCs regulate GSL biosynthesis not only by interacting with the GSL-MYBs but also due to their central 


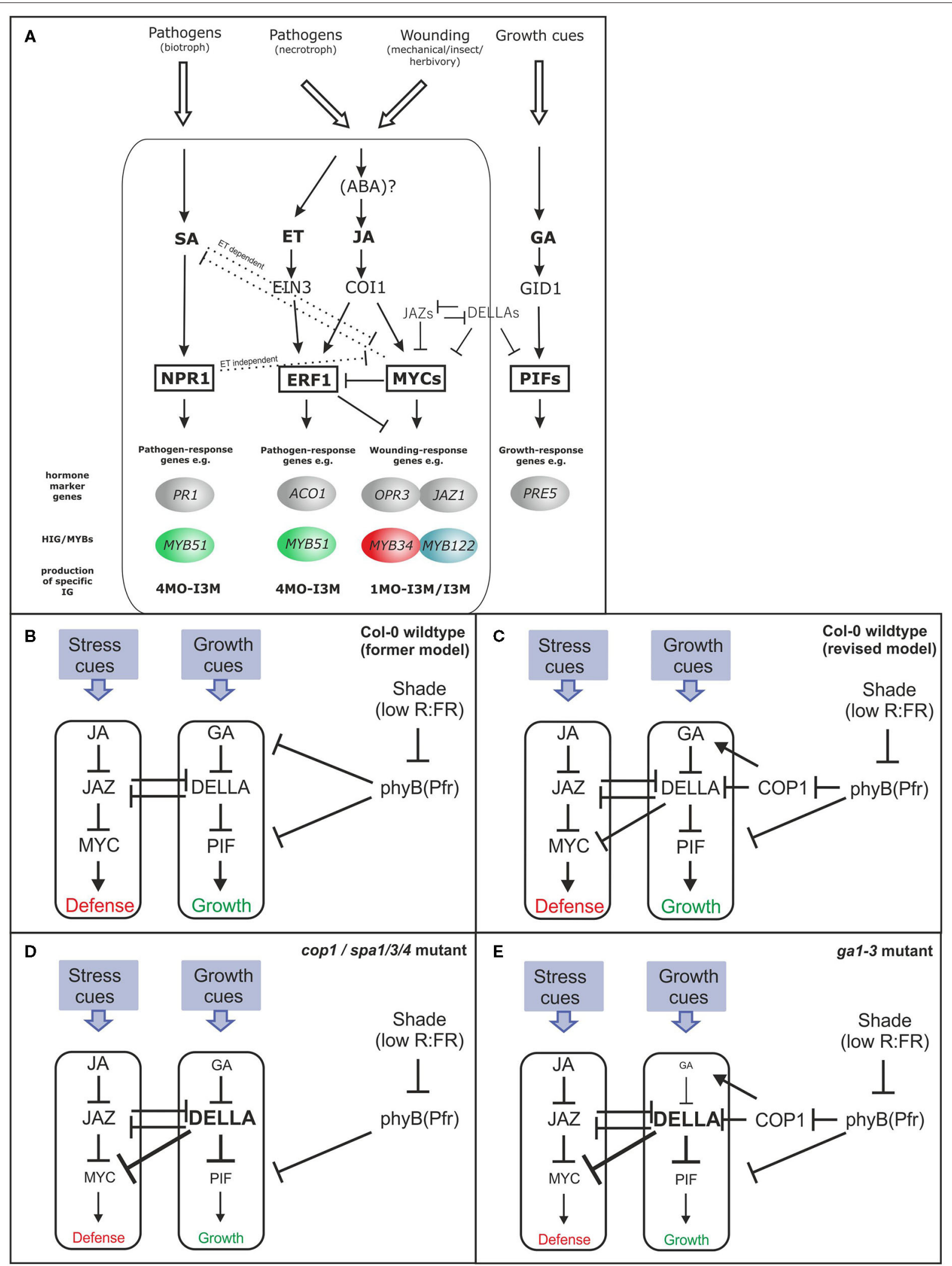

FIGURE 1 | Model for the hormonal signaling network in glucosinolate (GSL) regulation (A) and simplified model linking growth and defense trade-off (B-E). The figure depicts the postulated simplified hormonal signaling cascades in Arabidopsis thaliana in response to different pathogens involved in the regulation of the HIG-MYBs and the corresponding hormone marker genes. Different biotic/abiotic challenges activate different phytohormone signaling cascades and, thereby, a specific 
FIGURE 1 | combination of HIG/MYB transcription factors (TFs), which, in turn, led to the production of a specific mixture of IGs [altered from Frerigmann (2016)]. (B-E) Describes a simple model of the JA-GA signaling network that governs growth and defense (Campos et al., 2016) (B). In the updated model, COP1 is positioned between phyB and GA signaling. COP1 interacts with DELLA proteins and represses their activity in the dark and additionally affects the GA level probably via HY5 repression (Weller et al., 2009; Blanco-Touriñán et al., 2020) (C). These DELLA proteins positively affect JA signaling by interacting with JAZs, but the repression of MYCs is more relevant following strong DELLA accumulation in cop1, spa1/3/4 (D), or ga1-3 (E) as postulated in this study. (C) Provides a revised model in which overaccumulation of DELLAs in cop/spa mutants is responsible for the low GLS phenotype. This model contrasts the prevailing model (Navarro et al., 2008; Hou et al., 2010; Fernández-Calvo et al., 2011; Yang et al., 2012), in which JAZ-DELLA interactions cause reciprocal antagonism of GA and JA signaling. This updated model makes clear that the effects of JAZ-DELLA interaction can be overridden, when COP1/SPA regulates the DELLAs, affecting both the growth and biosynthesis of GSLs.

role in JA signaling and, thus, have the capability to activate the expression of IG-MYBs (Figure 1A; Frerigmann, 2016). Along with regulating GSLs, MYCs can also influence leaf development by interfering with the activity of, e.g., PIF4 and by promoting the activity of HY5 (Zhang et al., 2018; Ortigosa et al., 2020).

Thus, jazQ shows a specifically elevated level of JA-inducible GSLs such as glucobrassicin (I3M) (Campos et al., 2016), whereas the I3M level in $p h y B$ is lower (Cerrudo et al., 2017).

In this study, we identified that the COP1/SPA complex dramatically affects the accumulation of GSLs. This result suggests that the absence of the COP1/SPA complex can potentially increase DELLA protein abundance, which in turn, represses GA and JA signaling and inhibits MYC proteins. Low GSL phenotype of cop1 can be complemented by a dominant gain-of-function allele of MYC3 and by loss-offunction of DELLA proteins. Thus, COP1/SPA represents a crucial component that integrates light signaling in the hardwired hormone-linked transcriptional network and therefore regulates GSL biosynthesis under changing environmental conditions.

\section{MATERIALS AND METHODS}

\section{Plant Growth Conditions and Hormone Treatment}

Seeds of Arabidopsis thaliana mutant and respective wild-type (Table 1) were stratified for 2-7 days in the dark at $4^{\circ} \mathrm{C}$ to break seed dormancy. Plants were grown in growth cabinets in a light/dark cycle of $8 \mathrm{~h} / 16 \mathrm{~h}(9: 30-17: 30)$ and a day/night temperature of $21 / 18^{\circ} \mathrm{C}, 40 \%$ humidity, and a mean photon flux density of $120 \mu \mathrm{mol} \mathrm{m} \mathrm{m}^{-2} \mathrm{~s}^{-1}$. A minimum of $50 \mathrm{mg}$ of rosette material was harvested from 6-week-old plants in the middle of the day (13:30), was immediately frozen in liquid nitrogen, and kept at $-80^{\circ} \mathrm{C}$ until DNA and RNA extraction or GSL analysis.

For hormone treatment, wild-type and mutant plants were sprayed after 5.5 weeks with a MOCK, $50 \mu \mathrm{M} \mathrm{GA}_{3}, 50 \mu \mathrm{M}$ $\mathrm{MeJA}$, or $50 \mu \mathrm{M} \mathrm{MeJA} / 50 \mu \mathrm{M} \mathrm{GA}_{3}$ treatment. All treatments contained $0.02 \%$ Silwet to enhance the dispersion of the droplets onto the leaves. Plants were sprayed at three time points (10:30, $11: 30$, and 12:30) with the corresponding treatments and were kept separately under a hood to prevent cross-contamination. Rosette leaves for expression analysis were harvested $1 \mathrm{~h}$ after the last spray treatment (13:30). The remaining untreated pots were repeatedly sprayed once daily (10:30) with the hormone treatments and were harvested 3 days later at 13:30 (75 h after first spray treatment) for GSL determination.

\section{RNA Extraction and Quantitative Real-Time PCR Analysis}

Total RNA extraction and qRT-PCR analysis were performed as described by Frerigmann et al. (2012). The relative quantification of expression levels was performed using the comparative delta $\mathrm{Ct}$ method, and the calculated relative expression values were normalized to $P P 2 A$ and compared with the expression level in untreated wild-type plants $(\mathrm{Col}-0=1)$. If not specified in the figure legend, three independent experiments with three biological replicates from independently grown plants were analyzed (refer to Supplementary Table 1 for primer sequences).

\section{HPLC Analysis of Desulpho-GS}

The isolation and analysis of GSL content were performed using the desulpho-GSL method (Thies, 1979) on an ultra-performance liquid chromatography (UPLC) device (Waters, Eschborn) as described by Gigolashvili et al. (2012).

\section{Yeast Two-Hybrid Screen}

To identify SPA1 interactors, the REGIA yeast two-hybrid TF library (Paz-Ares et al., 2002) was screened using SPA1-pDEST32 as bait. The screen was performed as described by Maier et al. (2013).

\section{RESULTS}

\section{The COP1 and SPA Protein Complex Has a Pivotal Role in GSL Biosynthesis}

Previous studies have implicated MYB TFs of subgroup 12 (MYB34, MYB51, MYB122 and MYB28, MYB29, and MYB76) as central regulators of GSL biosynthesis (Gigolashvili et al., 2009; Sønderby et al., 2010; Frerigmann and Gigolashvili, 2014a). Furthermore, the complex signaling network upstream of MYBs includes TFs of higher hierarchical order, as well as a battery of different signaling cues and hormones, such as JA, SA, and ET (Figure 1A). It has also been shown that phyB has a central position in defining the balance of the growthdefense trade-off (Campos et al., 2016; Figure 1B). Because many phyB-mediated effects are transmitted via the action of the main repressor of light signaling, the COP1/SPA complex, this prompted an investigation on the role of this complex in the production of defense compounds. The accumulation of GSL defense compounds was analyzed in three mutants with defects in COP1 or SPA genes (cop1-4, cop1-6, and spa1/3/4). All three mutants showed a similar strong reduction of nearly $50 \%$ in AG and IG levels compared with the wild type (Figure 2A), 
TABLE 1 | Arabidopsis loss-of-function mutants used in this study.

\begin{tabular}{|c|c|c|c|c|}
\hline Mutant & Locus/ATG number & Description & Publication & Comment \\
\hline cop1-4 & COP1, AT2G32950 & EMS & Deng and Quail, 1992 & Weak allele \\
\hline cop1-6 & COP1, AT2G32950 & EMS & McNellis et al., 1994 & Weak allele \\
\hline atr2D & bHLH05/MYC3, AT5G46760 & EMS & Smolen et al., 2002 & Constitutively active MYC protein \\
\hline cop1-6 atr2D & & EMS & This work & \\
\hline ga1-3 & GA1, AT4G02780 & EMS & Koornneef and Van der Veen, 1980 & Reduced GA biosynthesis \\
\hline \multirow[t]{4}{*}{ spa1/3/4 } & & spa1-7 spa3-1 spa4-1 & Fittinghoff et al., 2006 & \\
\hline & SPA1, AT2G46340 & spa1-7 (SALK_023840) & Fittinghoff et al., 2006 & Full knockout \\
\hline & SPA3, AT3G15354 & spa3-1 (SAlL_569_F08) & Laubinger and Hoecker, 2003 & Full knockout \\
\hline & SPA4, AT1G53090 & spa4-1 (SAlL_590_C11) & Laubinger and Hoecker, 2003 & Full knockout \\
\hline gai-mimic & & pGREEN0179 & Willige et al., 2007 & Stable GAl protein \\
\hline \multirow[t]{3}{*}{ cop1-4/gai/rga } & & cop1-4 gai-td1 rga-29 & Blanco-Touriñán et al., 2020 & \\
\hline & GAI/RGA2, AT1G14920 & gai-td1 (SAlL_82_F06) & & \\
\hline & RGA1, AT2G01570 & rga-29 (SALK_089146) & & \\
\hline \multirow[t]{2}{*}{ ga1-3/rga/gai } & & gai-t6 & Achard et al., 2006 & \\
\hline & & rga-t2 & & \\
\hline \multirow[t]{2}{*}{ ga1-3/rga/gai/rg/1/rgl2 } & & $r g / 1-1$ & Achard et al., 2006 & \\
\hline & & rgl2-1 & & \\
\hline
\end{tabular}

highlighting a dramatic contribution of the COP/SPA complex to GSL production. The strong reduction in the expression of key marker genes (Figure 2B) for AG (CYP83A1) and IG biosynthesis (CYP79B3 and CYP83B1) indicated that the reduced biosynthesis of AG and IG could be associated with the low GSL phenotype in these mutants. The transcriptional regulators MYB34, MYB51, and MYB122 [High Indolic GSL(HIG)-MYB TFs] differentially integrate various signals to transcriptionally regulate GSL biosynthesis genes (Frerigmann and Gigolashvili, 2014a,b). Thus, these genes might have an altered expression pattern in copl and spa mutants and lead to reduced GSL biosynthesis. The main regulator MYB34 was transcriptionally downregulated at this developmental stage in cop1-4, cop16 , and spa1/3/4 compared with wild-type, whereas expression of MYB51 and MYB122 was upregulated (Figure 2C). The differential role of MYB34 vs. MYB51 and MYB122 in the regulation of IGs is a known phenomenon, which was reported before (Frerigmann and Gigolashvili, 2014a). MYB51 and MYB122 were observed to be induced when MYB34 levels are decreased, which seems to be a way to regulate the production and turnover of specific modified IG (Frerigmann et al., 2016).

\section{The cop1 and spa Mutants Possess a Reduced Expression of GSL Biosynthesis and JA Signaling Genes}

The reduced expression of JA-inducible MYB34 and elevated expression of ethylene- (ET) and SA-inducible MYB51 (Frerigmann and Gigolashvili, 2014a) in cop1 and spa mutants indicates a shift in the phytohormone response toward low JA and increased SA and/or ET signaling (Figure 1A). These HIG-MYB TFs interact with a group of MYC TFs to regulate GSL biosynthesis (Schweizer et al., 2013; Frerigmann et al., 2014). However, these MYC proteins (especially MYC2) are also crucial regulators of JA signaling (Fernández-Calvo et al., 2011). The expression of MYC2 and MYC4 was about $40 \%$ lower in cop14, cop1-6, and spa1/3/4 than in wild-type, suggesting that JA signaling was attenuated (Figure 2E). This was further confirmed by the reduced expression of the JA marker genes, JAZ1 and OPR3. Consistent with the known antagonistic crosstalk between JA and SA (Spoel et al., 2003), expression of the SA-marker gene PR1 was also highly induced (500-800-fold) in all three mutants, suggesting increased SA signaling (Figure 2D), as was expected due to high MYB51 expression.

Notably, the COP1/SPA complex was recently shown to interact with DELLA proteins in vivo, to ubiquitinate them and thereby negatively regulate the abundance of DELLA proteins in Arabidopsis in a light- and temperature-dependent manner (Blanco-Touriñán et al., 2020). Thus, the COP1/SPA-mediated degradation of DELLA is in line with observations made in this study, as the biosynthesis of GSLs is affected in cop and spa mutants (Figure 2).

In agreement with all these observations, the cop1-4, cop16 , and spa1/3/4 mutants showed strongly reduced $(\sim 80 \%)$ expression of the GA marker gene PRE5, substantiating the known role of DELLA proteins serving downstream of COP1 as key repressors of GA signaling (Figure 2D). The COP1/SPA complex is stabilized during darkness and ubiquitinates its targets in the nucleus (Hoecker, 2017). The abundance of DELLA proteins oscillates diurnally, with high levels in the afternoon/evening and low levels in darkness; however, imaging of GFP-tagged RGA expression in cop1-4 mutant seedlings indicated an altered oscillation pattern, with an increased abundance during darkness (Blanco-Touriñán et al., 2020). 


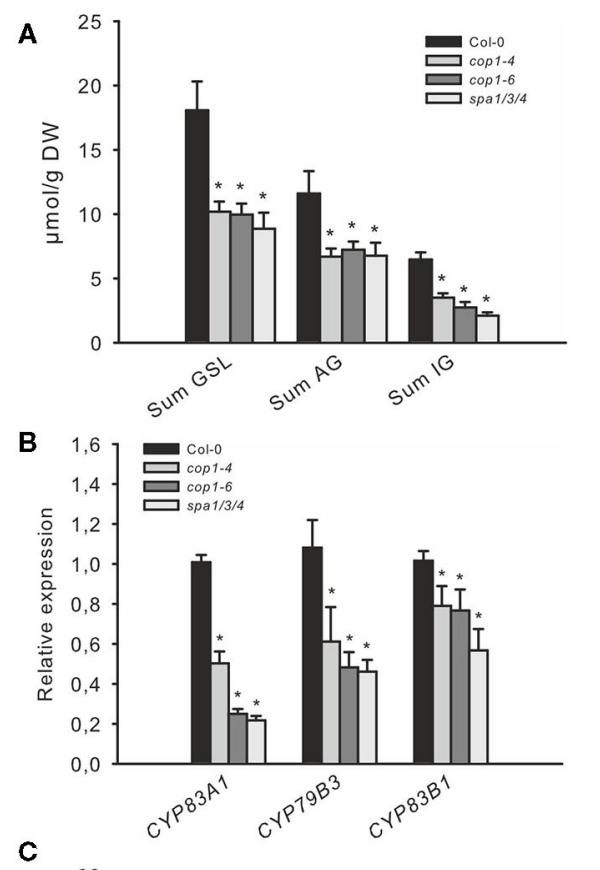

FIGURE 2 | of related genes (B-E), in leaves of short day (SD)-grown 6-week-old cop1and spa1/3/4 mutants. (A) The total contents of IGs and AGs, the four main aliphatic GSLs (3MSOP, 4MSOB, 5MSOP, and 8MSOO), and the three major indole GSLS (I3M, 4MO-I3M, and 1MO-I3M) are shown as "Sum GSLs," "Sum AG," and "Sum IG," respectively. Data are means \pm SE from four independent experiments with four biological replicates each $(n=$ 16). Values marked with asterisks differ significantly from Col-0 (Student's t-test; $p<0.05)$.

\section{GSL Accumulation Is Inhibited in ga1-3 and gai mimic Mutants}

The COP1/SPA complex affects many aspects of plant growth and development (Hoecker, 2017). To investigate whether the absence of COP1-dependent DELLA ubiquitination and the subsequently increased levels of DELLA proteins during the night are responsible for the observed low JA-signaling and the decreased production of GSL in cop1-4, cop1-6, and spa1/3/4, we analyzed two other mutants. The ga1-3 mutant is impaired in GA biosynthesis and thus accumulates DELLA proteins (Silverstone et al., 2001), whereas the gai mimic line overexpresses a dominant version of the DELLA protein GAI, which cannot interact with the GID1 receptor and thus accumulates in the cell (Willige et al., 2007). Both mutants, which are expected to accumulate DELLA, exhibited reduced AG and IG levels compared with the wild type and thus resembled the low GSL chemotype (Figure 3E) observed in cop1-4, cop1-6, and spa1/3/4 (Figure 2A). Similarly, both mutants showed the same expression level of GSL biosynthesis genes (Figure 3C), MYB34, MYB51, and MYB122 (Figure 3B); hormone marker genes (Figure 3C); and MYC genes (Figure 3D) as the copl and spa1/3/4 mutants (Figure 2). To further support the hypothesis that the DELLA proteins in gal-3 are responsible for the low GSL phenotype, we tested the ga1-3-complemented lines ga1-3/rga/gai and ga1-3/rga/gai/rgl1/2, which contain reduced DELLA levels due to the knockout of respective DELLAencoding genes (Figure 3F). These mutants exhibited partial complementation of the low GSL phenotype of ga 1-3 plants.

These results suggest that the absence of the COP1/SPA complex affects DELLA signaling in cells and represses the response of GA and JA. To test this hypothesis further, we reduced DELLA abundance by knocking out two out of the five DELLA proteins in the cop1-4 mutant. The absence of only two DELLA proteins was sufficient to partially complement the cop14 phenotype and led to larger leaves, more rosette leaves, and later flowering (Supplementary Figures 2, 3). Correspondingly, the low GSL chemotype in leaves of cop1-4 was partially complemented in cop1-4/rgal/gai (Figure 4A), mainly evident by an elevated AG level. Furthermore, expression of CYP79B3 was improved in this mutant (Figure 4B), without however significantly affecting the accumulation of IG levels. The absence of RGA1 and GAI in cop1-4 did not affect the expression of transcriptional regulators MYB34, MYB51, MYC3, and MYC4 and only slightly reduced MYB122 expression. Notably, the absence of only two out of the five DELLAs partially 


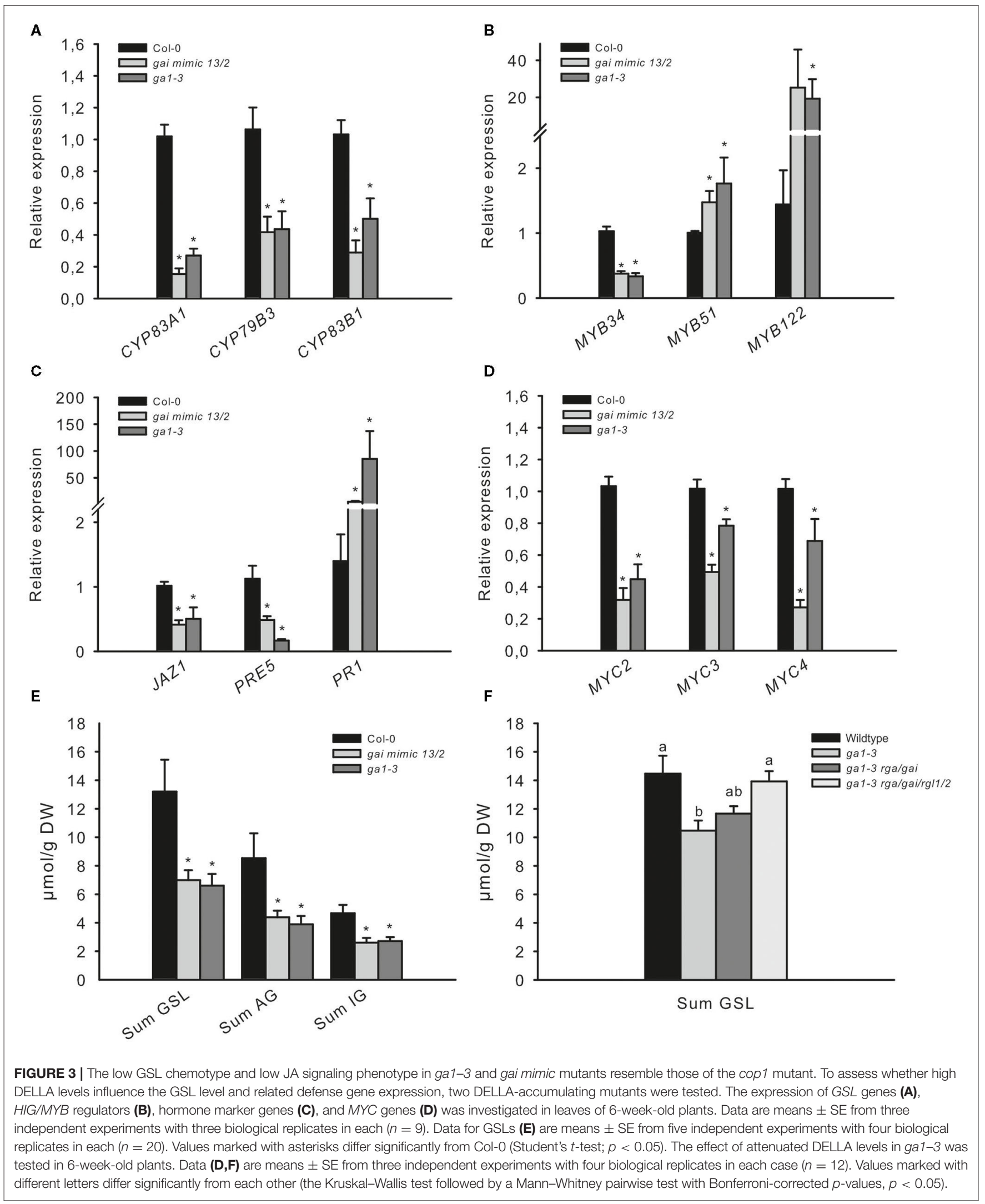


A
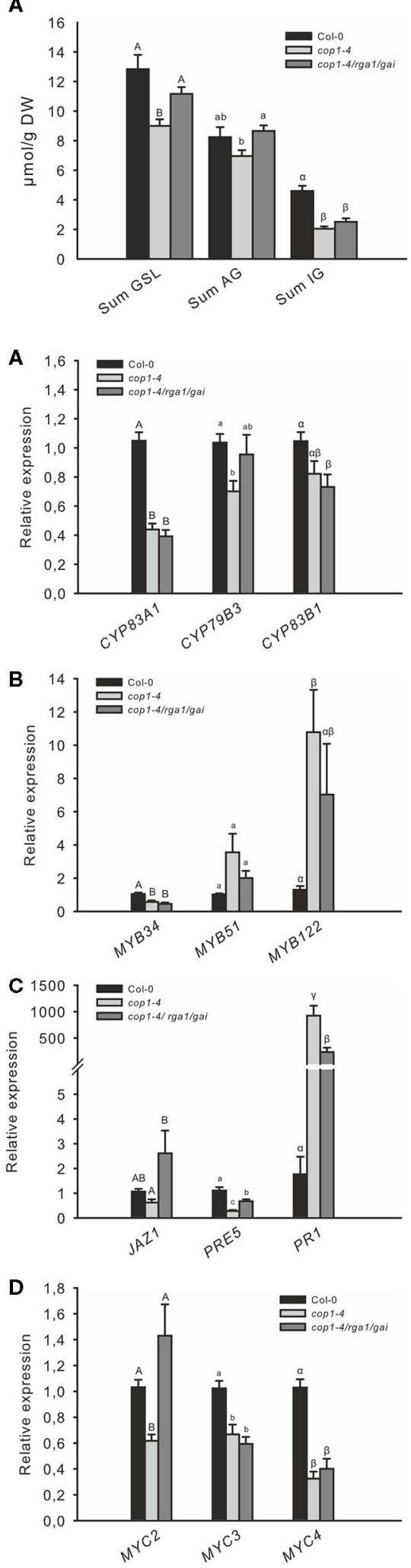

FIGURE 4 | The low GSL level of cop1 mutant is partially complemented by the absence of DELLAs. The absence of the two DELLA proteins, RGA1 and

(Continued)
FIGURE 4 | GAI, in cop1-4 affects the expression of GSL (A) GSL genes (B), HIG/MYB regulators (C), hormone marker genes (D), and MYC genes (E). Data for GSL levels (A) are means \pm SE from six independent experiments with four to six biological replicates in each case $(n=32)$. Expression data (B-E) are means \pm SE from three independent experiments with four biological replicates in each case $(n=12)$. Values marked with different letters differ significantly from each other (Kruskal-Wallis test followed by a Mann-Whitney pairwise test with Bonferroni-corrected $p$-values, $p<0.05$ ).

complemented the reduced expression of GA, JA, and SA marker genes as well as MYC2 in cop1-4 (Figures 4D,E). Significantly increased MYC2 mRNA levels in cop1-4/gai/rga vs. cop1-4 pointed to the substantial role of COP1/SPA in integrating hardwired JA/GA hormone-linked transcriptional network during GSL biosynthesis. Furthermore, this observation suggests that modulations in the DELLA pathway by COP1 knockout (cop1-4) resulted in low GA and JA response and high SA signaling.

\section{DELLAs Play a Central Role Within the JA/GA Hormonal Pathway During GSL Biosynthesis}

The JA and GA signaling pathways are important for the fine-tuning of the growth-defense trade-off: JA triggers the degradation of JAZ proteins and, in turn, de-represses plant defense, whereas GA treatment leads to the degradation of DELLA repressors and increased growth response. The DELLA and JAZ proteins physically interact and mutually repress functions of each other (Hou et al., 2010; Yang et al., 2012). Thus, following a single stress cue, two mechanisms act in concert to ensure that resources are appropriately allocated to either growth or defense. However, it is unclear which signal predominates if both stresses occur simultaneously. To analyze the effect of combined JA/GA treatment on IG biosynthesis, full-grown Col-0 plants were sprayed with these hormones individually or synchronously. Treatment with GA did not markedly affect transcript levels of IG biosynthesis genes or their transcriptional regulators, but JAZ1 and MYC2 (two out of the three tested JA marker genes) were slightly upregulated by GA (Figure 5). Treatment with JA strongly induced all tested IG biosynthesis genes, GSL regulators, and JA marker genes. Strikingly, combined GA and JA treatment downregulated most tested JA-inducible genes compared with JA treatment alone. This indicates that the induced degradation of DELLA proteins by GA might counteract activated JA signaling potentially due to the release of previously bound inactivated JAZ proteins, which, then, repress MYC proteins. It was similarly reported that the absence of four DELLAs in the dellaQ mutant leads to partial JA insensitivity (Navarro et al., 2008; Hou et al., 2010).

To further analyze the role of DELLAs on these hormonerelated gene expression changes, we similarly tested the GSL gene expression of ga1-3 in the response to JA and GA. Treatment with GA alone did not significantly affect the expression of JA-inducible genes (Figures 5A-F), but treatment with MeJA 

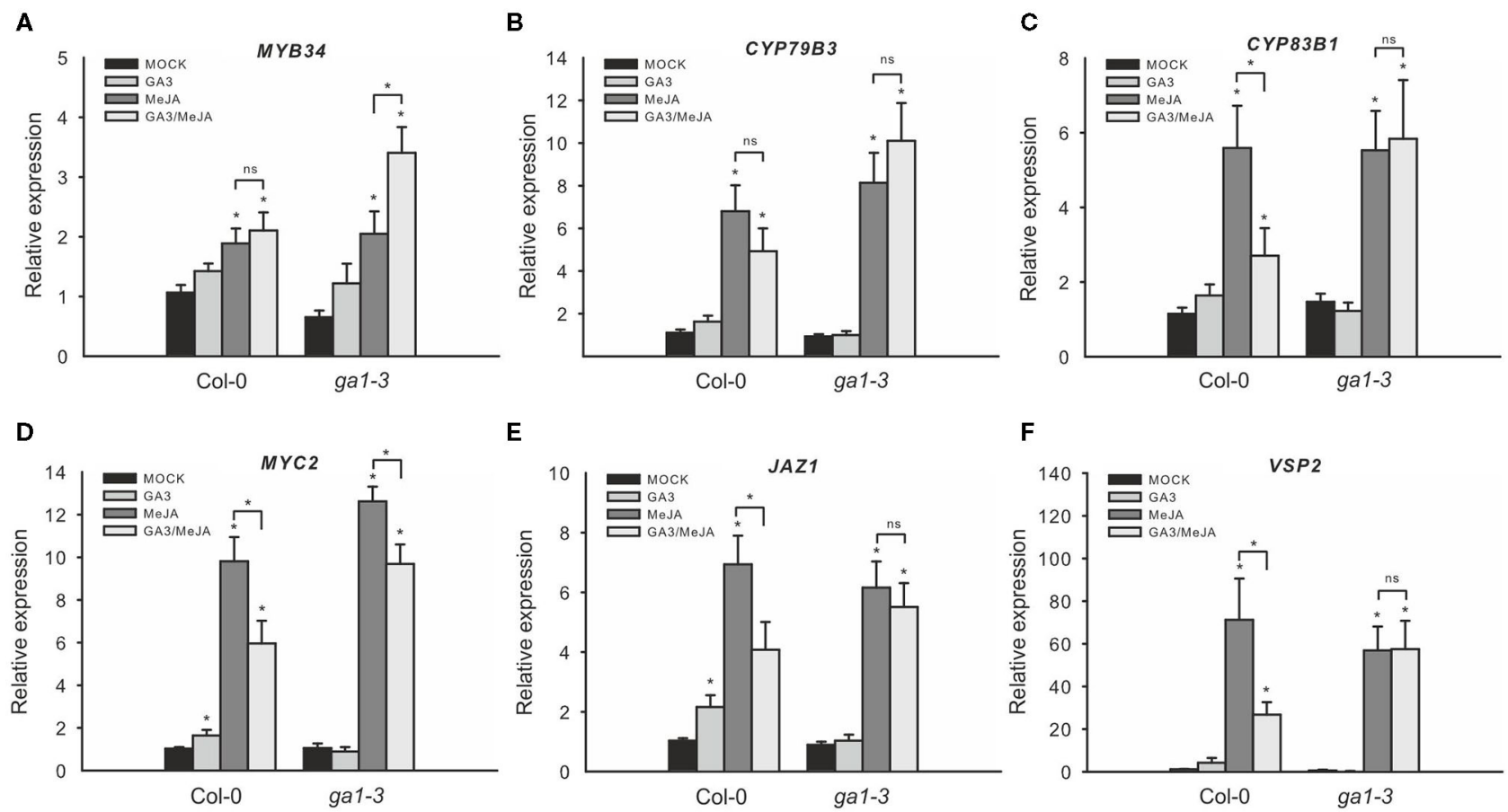

FIGURE 5 | Combined GAJJA treatment does not decrease IG biosynthesis gene expression in the ga1-3 mutant. The effect of combined GA/JA treatment on gene expression in the DELLA-accumulating mutant ga1-3 was tested in 6-week-old plants sprayed with MOCK, $50 \mu \mathrm{M}$ GA 3 , $50 \mu \mathrm{M}$ MeJA, or $50 \mu \mathrm{M}$ MeJA/50 $\mu \mathrm{M}$ GA 3 treatments. MYB34 (A), CYP79B3 (B), and CYP83B1 (C) served as marker genes for IG biosynthesis, whereas MYC2 (D), JAZ1 (E), and VSP2 (F) served as marker genes for JA signaling. Data are means \pm SE from three independent experiments with four biological replicates in each case $(n=12)$. Values marked with asterisks differ significantly from the respective MOCK treatment (Student's $t$-test; $p<0.05$ ). Values in parentheses indicate direct comparisons (ns, not significantly different; asterisks mark significantly different values; Student's $t$-test; $p<0.05$ ).

induced these genes to the same extent both in ga1-3 mutant and in Col-0. However, in contrast with Col-0, combined GA/MeJA treatment did not strongly reduce the expression of JA-inducible genes but even led to moderately increased expression of MYB34. This indicates that the degradation of DELLAs in ga 1-3 probably also releases other bound interaction partners, such as MYC proteins besides the JAZ proteins (Figure 1D).

The accumulation of GSLs in wild type was consistent with changes in observed gene expression. Treatment with GA did not affect GSL accumulation but JA treatment led to a strong increase in the level of GSL, especially IG (Figure 6). Although combined GA/JA treatment led to decreased IG gene expression than with JA alone, the IG levels were hardly lower than those in response to JA alone. The spa1/3/4 mutant did not respond by changing GSL accumulation after JA and GA treatments and only the IG content increased following GA/JA treatment. By contrast, the low GSL chemotype of ga1-3, whose phenotype results from high DELLA levels due to impaired GA biosynthesis, was partially complemented by GA treatment. Treatment with JA led to a greater IG induction and combined GA/JA treatment resulted in the highest IG level. The total IG level increased not significantly following GA/JA treatment; however, the level of I3M, which is JA-inducible, increased from 3.2 to $5.1 \mu \mathrm{mol} / \mathrm{g}$ DW. $4 \mathrm{MO}-\mathrm{I} 3 \mathrm{M}$, which is usually repressed upon JA, was decreased from 1.4 to $1.0 \mu \mathrm{mol} / \mathrm{g} \mathrm{DW}$
(Supplementary Figure 4). This increase was due to the GAinduced degradation of DELLA proteins since no increase was observed in the GA-insensitive DELLA-accumulating gai mimic mutant (Supplementary Figure 5).

To test the hypothesis of whether a high level of DELLA proteins caused MYC inactivation and might thus be responsible for the low GSL phenotype in such DELLA-accumulating mutants, we crossed the constitutively active MYC3 allele (atr2D) with cop1-6. The release of MYC3 from inactive complexes partially complemented the low GSL phenotype (Figure 7).

\section{DISCUSSION}

\section{The Production of GSL in Arabidopsis Is Modulated by the Interaction Between COP1/SPA and DELLA Proteins}

In this study, we investigated the role of COP1/SPA and DELLAs and identified them as new components in the regulation of GSL biosynthesis. Requirement of these upstream regulatory components seems to be inevitable upon dynamic environmental conditions as, among others, the production of GSL can be metabolically costly. Thus, flux balance analysis has estimated that the production of defense compounds requires a significant investment of energy and increases photosynthetic requirements by at least 15\% in Arabidopsis (Bekaert et al., 2012). Thus, studies 


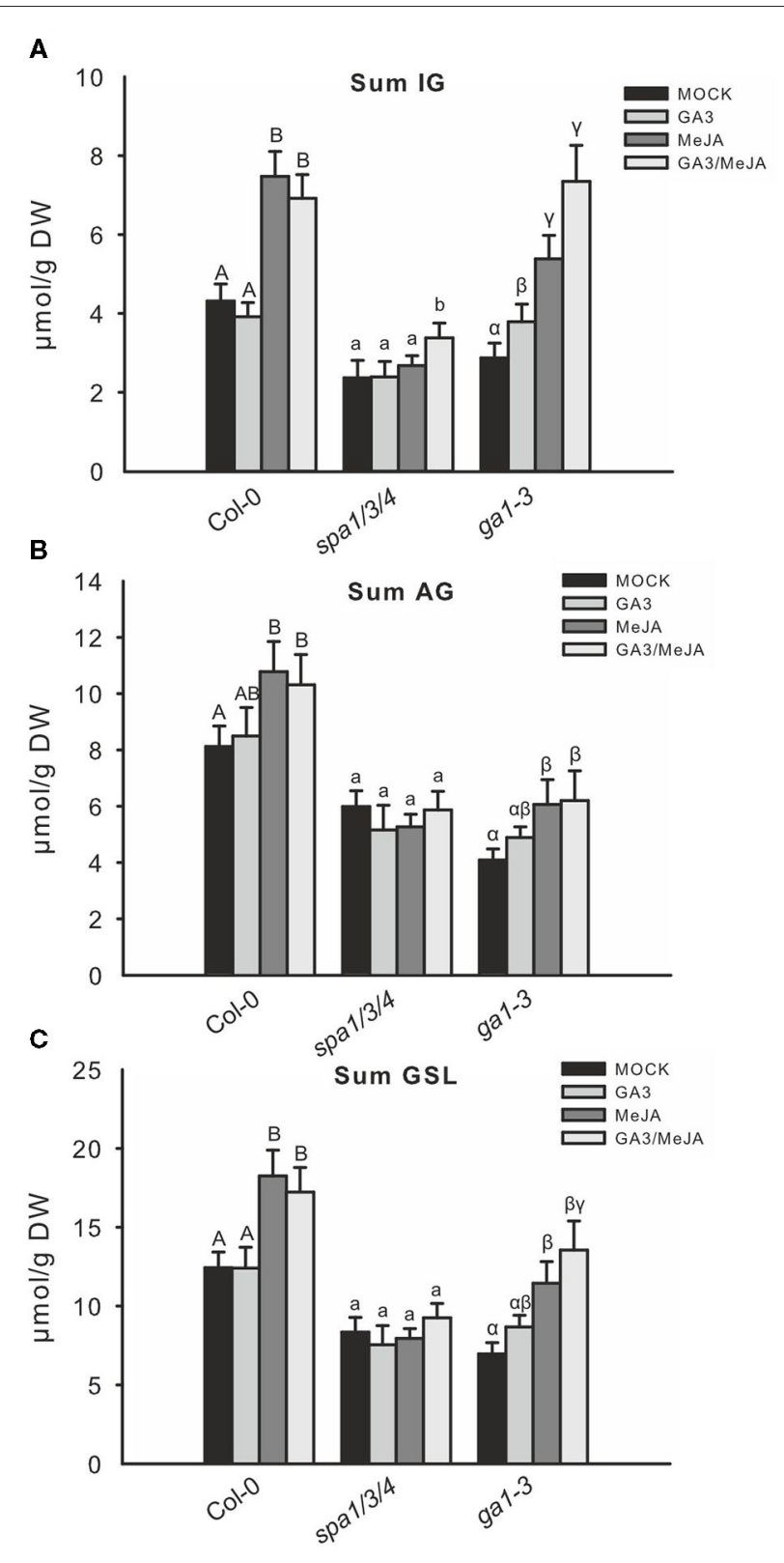

FIGURE 6 | Combined GA/JA treatment further increases the IG level in spa1/3/4 and ga1-3 mutants. The effect of combined GA/JA treatment on GSL accumulation was tested in 6-week-old spa1/3/4, ga1-3, and Col-0 wild-type plants sprayed with MOCK, $50 \mu \mathrm{M} \mathrm{GA}_{3}, 50 \mu \mathrm{M}$ MeJA, or $50 \mu \mathrm{M}$ MeJA/50 $\mu \mathrm{M} \mathrm{GA}_{3}$ treatments. Data $(\mathbf{A}-\mathbf{C})$ are means $\pm \mathrm{SE}$ from five independent experiments with four to six biological replicates in each case ( $n=25$ ). Different letters indicate significant differences (the Kruskal-Wallis test followed by a Mann-Whitney pairwise test with Bonferroni-corrected $p$-values, $p<0.05)$.

with Arabidopsis recombinant inbred lines (Paul-Victor et al., 2010) and with GSL knockout mutants at the early developmental stage (Züst et al., 2011) showed negative correlations between relative growth rates and higher GSL production. However, these effects disappeared during the plant life cycle, with a variable effect that depended on the type of GSL.

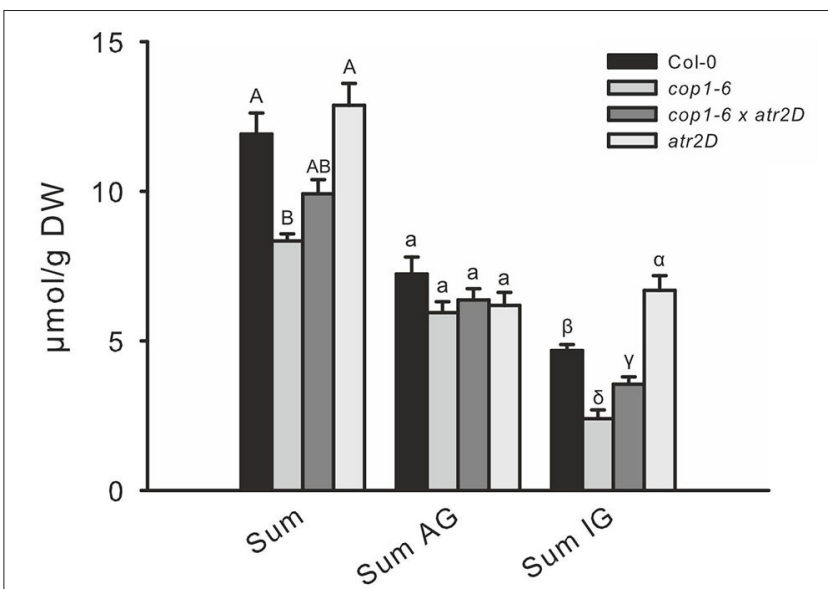

FIGURE 7 | The release of MYC3/ATR2 from inactive complexes partially complements low indole GSL phenotype of cop1. To release MYC3 from inactive complexes in cop1-6, the atr2D gain-of-function line was combined with cop1-6 to compare the GSL levels with that in cop1-6. Data are means \pm SE from four independent experiments with five or six biological replicates in each $(n=22)$. Values marked with different letters differ significantly from each other (the Kruskal-Wallis test followed by a Mann-Whitney pairwise test with Bonferroni-corrected $p$-values, $p<0.05$ ).

Molecular decisions made to coordinate growth and defense are determined by the abiotic environment including, in the case of GSLs, the availability of nutrients (Burow and Halkier, 2017), and also by light (Huseby et al., 2013). Light is an important signal for the allocation of plant resources to growth or defense. The absence of the main phyB photoreceptor in the jazQ mutant uncouples growth and defense responses by stimulating GA signaling and especially by activating PIF TFs (Campos et al., 2016). To investigate how GSL synthesis is affected by light and to uncover the molecular mechanism underlying phyB-dependent effects on the hormonal network for the growth-defense trade-off, we analyzed three different mutants in the COP1/SPA complex. Notably, all cop and spa mutants exhibited reduced growth (Supplementary Figure 2) and strongly reduced GSL synthesis (Figure 2A), which is consistent with reduced JA and GA signaling (Figures 2D,E). A yeast two-hybrid screen identified the DELLA protein GAI to be an interaction partner of SPA1 (Blanco-Touriñán et al., 2020), which could be confirmed by in vivo analysis. Arabidopsis DELLA proteins are key repressors of GA signaling and they serve as crucial convergence nodes of many signal transduction pathways that integrate hormonal responses and environmental stimuli (Claeys et al., 2014). The Arabidopsis genome encodes five DELLA proteins with distinct but redundant functions: GAI, RGA, RGL1, RGL2, and RGL3. In the absence of GA, DELLA proteins accumulate and repress GA responses, whereas, in the presence of GA, DELLA proteins are polyubiquitinated by the SCF ${ }^{\text {SLY1/GID2 }}$ complex and are subsequently degraded by the $26 \mathrm{~S}$ proteasome, thereby triggering GA signaling (Davière and Achard, 2013). Analogously, the interaction between the COP1/SPA complex and DELLA proteins can subsequently induce ubiquitination and degradation (Blanco-Touriñán et al., 
2020). During night or shade conditions, COP1 enters the nucleus and actively represses TFs via ubiquitination (Hoecker, 2017). The absence of COP1 thus causes an increase in DELLA abundance during the night (Blanco-Touriñán et al., 2020).

\section{Does Inactivation of COP1/SPA Complex Repress MYC Proteins and Reduce GSL Synthesis via DELLAs?}

The phenotype of other DELLA-accumulating mutants resembled that of cop1 mutants in terms of GSL accumulation and the expression of JA/GA marker-genes (Figure 3). DELLA proteins interact with and inhibit JAZ protein function (Hou et al., 2010) and JAZ proteins, in turn, interact with and inhibit MYC function. Thus, competition among DELLA proteins to interact with JAZs might lead to an increase in active MYC proteins, but the opposite regulation is observed in cop 1 and other DELLA-enriched mutants (Figures 2, 3). However, DELLA proteins additionally interact with MYC2 (Hong et al., 2012; Yazaki et al., 2016) and were shown to inhibit MYC2 function in the regulation of sesquiterpene biosynthesis. Therefore, JA and GA are necessary for the full activation of sesquiterpene biosynthesis (Hong et al., 2012). Correspondingly, the low GA and JA phenotype in cop1 and other DELLA-enriched mutants is presumably due to DELLA-inactivated bHLH TFs from the MYC and PIF subgroups.

Recent studies have shown that the three MYC proteins can be stabilized by red or blue light, whereas darkness and FR light promote their degradation (Chico et al., 2014). Furthermore, phytochromes and cryptochromes are required for MYC protein stability, and correspondingly, the destabilization of the MYC proteins in the dark is dependent on COP1. This suggests that despite lower MYC2 expression (Figure 2E; Supplementary Figure 6), the cop 1-4 mutant contains a higher level of MYC2 protein (Chico et al., 2014). Nevertheless, the expression of MYC-dependent target genes is strongly reduced in cop1 (Figures 2B-D; Supplementary Figure 6). This can be explained by the phenomenon known as "activation by destruction mechanism," which was previously reported for MYC2 (Zhai et al., 2013) and implies that MYC2 can function as a transcriptional activator if it undergoes turnover while it is phosphorylated and subsequently degraded. Therefore, the high level of MYC2 protein and the low JA signaling phenotype in cop1-4 can be explained by the absence of MYC protein activation and subsequent MYC degradation. Introduction of the dominant gain-of-function allele of MYC3 (atr2D) (Frerigmann et al., 2014; Goossens et al., 2015) into the cop1-6 background largely rescued the low IG phenotype (Figure 7), pointing that the gain-of-function of MYC3, which can potentially enforce the accumulation of active MYC3 protein, is capable of rescuing the low GSL chemotype of cop1 mutants.

Similarly, a reduction in the level of DELLA proteins in cop1-4 in the absence of $R G A / G A I$ rescued the low JA and GA phenotype partially (Figure 4; Supplementary Figure 3). Furthermore, the low degree of GSL and JA signaling in the DELLA-enriched mutant ga1-3 was mostly rescued by the absence of four DELLA proteins (Figure 3), as shown by the expression of JA marker genes (PDF1-2, LOX2, and TAT1) in the $\mathrm{ga} 1-3 / \mathrm{rga} / \mathrm{gai} / \mathrm{rgl} / \mathrm{rgl} 2$ mutant (Hou et al., 2010).

\section{Light Perception and the Induction of Secondary Defense Compounds Are Linked to a Higher Level of DELLA Proteins and MYC Inactivation}

In this study, we propose that light-activated photoreceptors inhibit the function of the COP1/SPA complex, which subsequently leads to higher levels of DELLA proteins; these DELLA proteins, in turn, inhibit and stabilize MYCs proteins by physical interaction (Figure 1C). This model is an add-on to the prevailing one as reported in previous studies (Navarro et al., 2008; Hou et al., 2010; Yang et al., 2012; Huot et al., 2014), in which JAZ-DELLA interactions cause reciprocal antagonism of GA and JA signaling. The updated model in Figure 1C makes clear that the effects of JAZ-DELLA interaction can be overridden, when COP1/SPA regulates the DELLAs, affecting in this way both the growth as well as biosynthesis of GSLs. This hypothesis is supported by observations that transferring plants from short days (SD) to long days (LD) increases resistance to Botrytis cinerea (Cagnola et al., 2018). The defensive secondary compounds required for resistance against this necrotrophic pathogen include camalexin and other indolic-derived secondary compounds, which are synthesized similarly to the IGs addressed in this study. The perception of a longer light period, which activates the synthesis of these compounds and results in improved plant resistance, is dependent on the photoreceptors phyA, cry1, and cry2, whereas exposure to short days decreases MYC2 stability in a COP1dependent manner. Correspondingly, a longer light period inhibits the COP1/SPA complex and increases nuclear DELLA abundance (Cagnola et al., 2018). Thus, DELLA proteins interact with, inhibit, and stabilize MYC2. Accordingly myc2 and cop1 mutants show enhanced resistance to $B$. cinerea in SD (Cagnola et al., 2018). Contrary to the increased $B$. cinerea resistance following inactivation of COP1 and MYC2 by LD (compared with SD), shade conditions (FR light treatment) increased susceptibility to $B$. cinerea (Cerrudo et al., 2012) by triggering the inactivation of phyB (a COP1/SPA repressor). Therefore, the phyB mutant was highly susceptible to $B$. cinerea without FR treatment (Cerrudo et al., 2012). FR light and phyB mutation trigger DELLA degradation and increase JAZ10 stability (Leone et al., 2014), which is consistent with a higher activity of the COP1/SPA complex.

Exposure to FR light increases susceptibility to $B$. cinerea due to reduced JA sensitivity, resulting in lower IG and camalexin levels, and this is additionally controlled by JAZ10, because the jaz10 loss-of-function mutant lacks FR lightdependent reduction in IG in normal conditions and following MeJA treatment (Cargnel et al., 2014). Furthermore, the stability of most JAZ proteins is enhanced by FR light, which delays JA-dependent JAZ degradation (Chico et al., 2014). However, the absence of JAZ10 function appears to repress many of the defense phenotypes in the phyB mutant, such as low IG content, high susceptibility to $B$. 
cinerea, and low JA-inducible gene expression, but not the shade avoidance phenotypes (Cerrudo et al., 2017). This indicates that a low level of DELLA proteins together with decreased abundance of JAZ proteins might not strongly repress GA signaling, including shade-avoidance responses by PIF proteins.

\section{The Ratio of DELLA-MYC, DELLA-JAZ, and JAZ-MYC Inactive Complexes Might Depend on the Relative Different Stoichiometric Abundances of Their Components in Diverse Tissues and Following Biotic/Abiotic Stimuli}

The degradation of DELLA proteins in the presence of GA releases JAZ proteins to attenuate MYC2 function (Hou et al., 2010). Combined treatment with MeJA and GA strongly reduced the expression of JA-induced genes compared to treatment with JA alone (Figure 5; Supplementary Figure 7). However, treatment with GA alone did not repress these genes. The production of GSLs, especially IG, was strongly induced by JA but was hardly affected by combined GA/JA treatment or GA alone. However, treatment of the DELLAaccumulating mutant, ga1-3, with GA increased the level of JA-inducible IG (Figure 6; Supplementary Figure 7), indicating that the resulting decrease in DELLA protein abundance releases MYC proteins from inactive complexes. Similarly, combined JA/GA treatment did not lead to a reduction in GSL content compared with JA treatment alone, as in Col-0, but the content of the JA-inducible IG, I3M, increased strongly (Supplementary Figure 4). Compared to the reduced expression of JA-inducible genes in Col-0, combined GA/JA treatment led to a slight increase or at least stable transcript levels of most JA-inducible GSL regulatory or synthesis genes in gal3 (Figure 5). Thus, in a DELLA-enriched mutant, the GAinduced degradation of DELLA proteins in addition to the JAinduced degradation of JAZ proteins might release even more MYC proteins from inactive complexes than JA treatment alone. Similarly, the spa1/3/4, which accumulates DELLA proteins, showed a higher content of IG following combined JA/GA treatment compared with treatment with JA alone (Figure 6; Supplementary Figure 7). The greater response of gal-3 than spa1/3/4 to combined GA/JA treatment indicates that the COP1/SPA complex might affect GA and JA signaling in a much more complex manner. For example, MYC2-mediated abscisic acid (ABA) and JA responses are further modulated by SPA1 (Gangappa et al., 2010), and JA stabilizes several COP1-targeted TFs in a COP1-dependent manner (Zheng et al., 2017). Another study has identified a sulfotransferase (ST2a) recently and showed that it is strongly upregulated by plant proximity perceived by phyB (Fernández-Milmanda et al., 2020). By catalyzing the formation of a sulfated JA derivative, ST2a acts to degrade bioactive forms of JA and represents another molecular link between photoreceptors and hormone signaling in plants.

\section{CONCLUSIONS}

We have uncovered another crucial component of the hardwired hormone-linked transcriptional network upstream from the MYC-bHLH TF complex, which regulates the production of defensive GSL compounds (Figure 1; Supplementary Figure 7). The COP1/SPA complex is a central nexus that integrates light perception via phyB and other photoreceptors and transforms several interconnected components into a finely balanced system of growth and defense. The COP1/SPA complex positively regulates growth at an additional level via interacting with and ubiquitinating DELLA proteins in shade or night conditions (Blanco-Touriñán et al., 2020).

The COP1/SPA complex also interacts with PIF proteins (Zhu et al., 2015) and is critical to maintaining high GA levels in varying light conditions (Weller et al., 2009). The DELLA proteins themselves constitute a crucial convergence point of many signal transduction pathways that are triggered by hormones and environmental stimuli (Claeys et al., 2014). We show that different DELLA-accumulating mutants not only inhibit JAZ repressors but strongly repress general JA signaling via MYC interaction (Figure 1; Supplementary Figure 7). GSL synthesis can only be fully activated by simultaneous activation of GA and JA pathways, a situation that resembles the induction of sesquiterpene biosynthesis in inflorescences (Hong et al., 2012). Inflorescences also possess a high level of DELLA protein expression (Lee et al., 2002; Tyler et al., 2004). Thus, the observed inactivation of MYC proteins by DELLA proteins appears to represent an additional layer of regulation that depends on the stoichiometric ratio of DELLA and JAZ proteins in specific tissues or cells. Such a multi-tiered mechanism to powerfully repress the expression of chemical defenses might prevent deleterious effects of carbon depletion or metabolic imbalance during the growth-to-defense transition (Guo et al., 2018). In specific tissues and situations that led to a high abundance of DELLA proteins, this additional layer of regulation might safeguard against too many resources being allocated to defense in the absence of a specific trigger. Overall, independent of molecular mechanisms behind growth-defense trade-off under given environmental conditions, the whole ontogeny of the organisms and their environmental context needs to be taken into account. The production of defense compounds does not necessarily incur net ecological costs, because the metabolic requirements for defense are not automatically the same as for those for growth (Kliebenstein, 2016; Mitreiter and Gigolashvili, 2021).

In summary, these findings highlight the importance of COP1/SPA and DELLA upstream regulatory components in balancing the defense and growth. However, growth restriction does not seem to be a direct consequence of defense activation but rather a part of a defense system, which is adaptive under conditions of a biotic attack and maladaptive under conditions where defense is not the major functional priority for survival (BallaréBallaré and Austin, 2019). The complexity of this pathway is also highlighted in contrasting observations made during the analysis of phy jaz mutants. While phyB jazQ plants capable of uncoupling growth and defense as PIF and MYC 
TFs are getting simultaneously activated (Campos et al., 2016), the phyB jazD is unable to completely uncouple growth-defense phenotypes (Major et al., 2020). Notably, the abundant RGA protein was not increased in untreated $j a z Q$ or jazD seedlings relative to WT (Major et al., 2020), suggesting that either (i) change in DELLA protein activity does not play a major role in restricting the shoot growth of jaz mutants or (ii) that the DELLA pathway (e.g., yet to be explored regulatory RNAs or small signaling metabolites) is involved in this process but not the DELLA proteins.

Thus, the molecular mechanism of JA-induced biomass reduction and the potential role of DELLAs and MYCs in this process continues to be thrilling. Not only the role of the DELLA pathway beyond its activity on protein level but also the role of MYCs in the regulation of plant growth-defense balance needs to be addressed in the future in more detail. Exploration of other signaling options, including small regulatory RNA and metabolite signals, is a must to find the solution for growthdefense conflicts in plants. While progress has been made to identify some hard-wired genetic and ecophysiological regulators that constrain plant growth and development during immune responses, recent advances in disciplines like systems biology, bioinformatics, and machine learning will open the gate for the identification of missing regulatory mechanisms balancing the plant growth and immunity.

\section{DATA AVAILABILITY STATEMENT}

The original contributions presented in the study are included in the article/Supplementary Material, further inquiries can be directed to the corresponding author/s.

\section{REFERENCES}

Achard, P., Cheng, H., De Grauwe, L., Decat, J., Schoutteten, H., Moritz, T., et al. (2006). Integration of plant responses to environmentally activated phytohormonal signals. Science 311, 91-94. doi: 10.1126/science.1118642

Ballaré, C. L., and Austin, A. T. (2019). Recalculating growth and defense strategies under competition: key roles of photoreceptors and jasmonates. J. Exp. Bot. 70, 3425-3434. doi: 10.1093/jxb/erz237

Bekaert, M., Edger, P. P., Hudson, C. M., Pires, J. C., and Conant, G. C. (2012). Metabolic and evolutionary costs of herbivory defense: systems biology of glucosinolate synthesis. New Phytol. 196, 596-605. doi: 10.1111/j.1469-8137.2012.04302.x

Blanco-Touriñán, N., Legris, M., Minguet, E. G., Costigliolo-Rojas, C., Nohales, M. A., Iniesto, E., et al. (2020). COP1 destabilizes DELLA proteins in Arabidopsis. Proc. Natl. Acad. Sci. 117, 13792-13799. doi: 10.1073/pnas.1907969117

Burow, M. (2016). Complex environments interact with plant development to shape glucosinolate profiles. Adv. Bot. Res. 80, 15-30. doi: 10.1016/bs.abr.2016.06.001

Burow, M., and Halkier, B. A. (2017). How does a plant orchestrate defense in time and space? using glucosinolates in Arabidopsis as case study. Curr. Opin. Plant Biol. 38, 142-147. doi: 10.1016/j.pbi.2017.04.009

Cagnola, J. I., Cerdan, P. D., Pacin, M., Andrade, A., Rodriguez, M. V., Zurbriggen, M., et al. (2018). Long-day photoperiod enhances jasmonic acid-related plant defense. Plant Physiol. 178, 163-173. doi: 10.1104/pp.18.00443

Campos, M. L., Yoshida, Y., Major, I. T., de Oliveira Ferreira, D., Weraduwage, S. M., Froehlich, J. E., et al. (2016). Rewiring of jasmonate and phytochrome B signalling uncouples plant growth-defense tradeoffs. Nat. Commun. 7:12570. doi: $10.1038 /$ ncomms 12570

\section{AUTHOR CONTRIBUTIONS}

HF designed the experiments, performed most of the experiments, analyzed the data, and wrote the manuscript. TG performed qPCR experiments and was actively involved in the writing and publication process. $\mathrm{UH}$ contributed to the experimental design and the writing. All authors contributed to the article and approved the submitted version.

\section{FUNDING}

This study was supported by the Deutsche Forschungsgemeinschaft: Project Reference Numbers: EXC 1028 and GI 824/3-1.

\section{ACKNOWLEDGMENTS}

We thank Ulrike Hebbeker (University of Cologne) and Anna Lisa Roth (Max Planck Institute for Plant Breeding Research) for technical assistance; Claus Schwechheimer, Patrick Achard, and David Alabadi for kindly providing seeds; and Jorge Casal, John Chandler, and Paul Schulze-Lefert for critically reading the manuscript.

\section{SUPPLEMENTARY MATERIAL}

The Supplementary Material for this article can be found online at: https://www.frontiersin.org/articles/10.3389/fpls.2021. 680255/full\#supplementary-material

Cargnel, M. D., Demkura, P. V., and Ballaré, C. L. (2014). Linking phytochrome to plant immunity: low red: far:red ratios increase Arabidopsis susceptibility to Botrytis cinerea by reducing the biosynthesis of indolic glucosinolates and camalexin. New Phytol. 204, 342-354. doi: 10.1111/nph. 13032

Casal, J. J. (2013). Photoreceptor signaling networks in plant responses to shade. Annu. Rev. Plant Biol. 64, 403-427. doi: 10.1146/annurev-arplant-050312-120221

Cerrudo, I., Caliri-Ortiz, M. E., Keller, M. M., Degano, M. E., Demkura, P. V., and Ballaré, C. L. (2017). Exploring growth-defence trade-offs in Arabidopsis: phytochrome B inactivation requires JAZ10 to suppress plant immunity but not to trigger shade-avoidance responses. Plant Cell Environ. 40, 635-644. doi: $10.1111 /$ pce. 12877

Cerrudo, I., Keller, M. M., Cargnel, M. D., Demkura, P. V., de Wit, M., Patitucci, M. S., et al. (2012). Low red/far-red ratios reduce Arabidopsis resistance to Botrytis cinerea and jasmonate responses via a COI1JAZ10-dependent, salicylic acid-independent mechanism. Plant Physiol. 158, 2042-2052. doi: 10.1104/pp.112.193359

Chico, J.-M., Fernández-Barbero, G., Chini, A., Fernández-Calvo, P., Díez-Díaz, M., and Solano, R. (2014). Repression of jasmonate-dependent defenses by shade involves differential regulation of protein stability of MYC transcription factors and their JAZ repressors in Arabidopsis. Plant Cell 26, 1967-1980. doi: 10.1105/tpc.114.125047

Claeys, H., De Bodt, S., and Inzé, D. (2014). Gibberellins and DELLAs: central nodes in growth regulatory networks. Trends Plant Sci. 19, 231-239. doi: 10.1016/j.tplants.2013.10.001

Davière, J.-M., and Achard, P. (2013). Gibberellin signaling in plants. Development 140, 1147-1151. doi: 10.1242/dev.087650 
Deng, X. W., and Quail, P. H. (1992). Genetic and phenotypic characterization of cop1 mutants of Arabidopsis thaliana. Plant J. 2, 83-95. doi: 10.1111/j.1365-313X.1992.00083.x

Feng, S., Martinez, C., Gusmaroli, G., Wang, Y., Zhou, J., Wang, F., et al. (2008). Coordinated regulation of Arabidopsisthaliana development by light and gibberellins. Nature 451:475. doi: 10.1038/nature06448

Fernández-Calvo, P., Chini, A., Fernández-Barbero, G., Chico, J.-M., GimenezIbanez, S., Geerinck, J., et al. (2011). The Arabidopsis bHLH transcription factors MYC3 and MYC4 are targets of JAZ repressors and act additively with MYC2 in the activation of jasmonate responses. Plant Cell 23, 701-715. doi: $10.1105 /$ tpc. 110.080788

Fernández-Milmanda, G. L., Crocco, C. D., Reichelt, M. Mazza C. A., Köllner, T. G., Zhang, T. et al. (2020). A light-dependent molecular link between competition cues and defence responses in plants. Nat. Plants 6, 223-230. doi: 10.1038/s41477-020-0604-8

Fittinghoff, K., Laubinger, S., Nixdorf, M., Fackendahl, P., Baumgardt, R. L., Batschauer, A., et al. (2006). Functional and expression analysis of Arabidopsis SPA genes during seedling photomorphogenesis and adult growth. Plant J. 47, 577-590. doi: 10.1111/j.1365-313X.2006.02812.x

Frerigmann, H. (2016). Glucosinolate regulation in a complex relationship-MYC and MYB-no one can act without each other. Adv. Bot. Res. 80, 57-97. doi: 10.1016/bs.abr.2016.06.005

Frerigmann, H., Berger, B., and Gigolashvili, T. (2014). bHLH05 is an interaction partner of MYB51 and a novel regulator of glucosinolate biosynthesis in Arabidopsis. Plant Physiol. 166, 349-369. doi: 10.1104/pp.114.240887

Frerigmann, H., Böttcher, C., Baatout, D., and Gigolashvili, T. (2012). Glucosinolates are produced in trichomes of Arabidopsis thaliana. Front. Plant Sci. 3:242. doi: 10.3389/fpls.2012.00242

Frerigmann, H., and Gigolashvili, T. (2014a). MYB34, MYB51, and MYB122 distinctly regulate indolic glucosinolate biosynthesis in Arabidopsis thaliana. Mol. Plant 7, 814-828. doi: 10.1093/mp/ssu004

Frerigmann, H., and Gigolashvili, T. (2014b). Update on the role of R2R3-MYBs in the regulation of glucosinolates upon sulfur deficiency. Front. Plant Sci. 5:626. doi: $10.3389 /$ fpls.2014.00626

Frerigmann, H., Piślewska-Bednarek, M., Molina, A., Sánchez-Vallet, A., Glawischnig, E., Gigolashvili, T., et al. (2016). MYB transcription factors in regulation of pathogen triggered tryptophan metabolism in Arabidopsis thaliana. Mol. Plant. 9, 682-695. doi: 10.1016/j.molp.2016.01.006

Gangappa, S. N., Prasad, V. B. R., and Chattopadhyay, S. (2010). Functional interconnection of MYC2 and SPA1 in the photomorphogenic seedling development of Arabidopsis. Plant Physiol. 154, 1210-1219. doi: 10.1104/pp.110.163717

Gigolashvili, T., Berger, B., and Flügge, U. I. (2009). Specific and coordinated control of indolic and aliphatic glucosinolate biosynthesis by R2R3-MYB transcription factors in Arabidopsis thaliana. Phytochem. Rev. 8, 3-13. doi: 10.1007/s11101-008-9112-6

Gigolashvili, T., Geier, M., Ashykhmina, N., Frerigmann, H., Wulfert, S., Krueger, S., et al. (2012). The Arabidopsis thylakoid ADP/ATP carrier TAAC has an additional role in supplying plastidic phosphoadenosine 5 -phosphosulfate to the cytosol. Plant Cell 24, 4187-4204. doi: 10.1105/tpc.112.101964

Goossens, J., Swinnen, G., Vanden Bossche, R., Pauwels, L., and Goossens, A. (2015). Change of a conserved amino acid in the MYC2 and MYC3 transcription factors leads to release of JAZ repression and increased activity. New Phytol. 206, 1229-1237. doi: 10.1111/nph.13398

Guo, Q., Major, I. T., and Howe, G. A. (2018). Resolution of growth-defense conflict: mechanistic insights from jasmonate signaling. Curr. Opin. Plant Biol. 44, 72-81. doi: 10.1016/j.pbi.2018.02.009

Hoecker, U. (2017). The activities of the E3 ubiquitin ligase COP1/SPA, a key repressor in light signaling. Curr. Opin. Plant Biol. 37, 63-69. doi: 10.1016/j.pbi.2017.03.015

Hong, G.-J., Xue, X.-Y., Mao, Y.-B., Wang, L.-J., and Chen, X.-Y. (2012). Arabidopsis MYC2 interacts with DELLA proteins in regulating sesquiterpene synthase gene expression. Plant Cell 24, 2635-2648. doi: 10.1105/tpc.112.098749

Hou, X., Ding, L., and Yu, H. (2013). Crosstalk between GA and JA signaling mediates plant growth and defense. Plant Cell Rep. 32, 1067-1074. doi: 10.1007/s00299-013-1423-4
Hou, X., Lee, L. Y. C., Xia, K., Yan, Y., and Yu, H. (2010). DELLAs modulate jasmonate signaling via competitive binding to JAZs. Dev. Cell 19, 884-894. doi: 10.1016/j.devcel.2010.10.024

Howe, G. A., Major, I. T., and Koo, A. J. (2018). Modularity in jasmonate signaling for multistress resilience. Annu. Rev. Plant Biol. 69, 387-415. doi: 10.1146/annurev-arplant-042817-040047

Huot, B., Yao, J., Montgomery, B. L., and He, S. Y., (2014). Growth-defense tradeoffs in plants: a balancing act to optimize fitness. Mol Plant.7:1267-1287. doi: $10.1093 / \mathrm{mp} / \mathrm{ssu} 049$

Huseby, S., Koprivova, A., Lee, B.-R., Saha, S., Mithen, R., Wold, A.-B., et al. (2013). Diurnal and light regulation of sulphur assimilation and glucosinolate biosynthesis in Arabidopsis. J. Exp. Bot. 64, 1039-1048. doi: 10.1093/jxb/ers378

Kliebenstein, D. J. (2016). False idolatry of the mythical growth versus immunity tradeoff in molecular systems plant pathology. Physiol. Mol. Plant Pathol. 95, 55-59. doi: 10.1016/j.pmpp.2016.02.004

Koornneef, M., and Van der Veen, J. (1980). Induction and analysis of gibberellin sensitive mutants in Arabidopsis thaliana (L.) Heynh. Theor. Appl. Genet. 58, 257-263. doi: 10.1007/BF00265176

Laubinger, S., and Hoecker, U. (2003). The SPA1-like proteins SPA3 and SPA4 repress photomorphogenesis in the light. Plant J. 35, 373-385. doi: 10.1046/j.1365-313X.2003.01813.x

Lee, S., Cheng, H., King, K. E., Wang, W., He, Y., Hussain, A., et al. (2002). Gibberellin regulates Arabidopsis seed germination via RGL2, a GAI/RGA-like gene whose expression is up-regulated following imbibition. Genes Dev. 16, 646-658. doi: 10.1101/gad.969002

Leone, M., Keller, M. M., Cerrudo, I., and Ballar,é C. L. (2014). To grow or defend? low red: far-red ratios reduce jasmonate sensitivity in Arabidopsis seedlings by promoting DELLA degradation and increasing JAZ10 stability. New Phytol. 204, 355-367. doi: 10.1111/nph.12971

Maier, A., Schrader, A., Kokkelink, L., Falke, C., Welter, B., Iniesto, E., et al. (2013). Light and the E3 ubiquitin ligase COP1/SPA control the protein stability of the MYB transcription factors PAP1 and PAP2 involved in anthocyanin accumulation in Arabidopsis. Plant J. 74, 638-651. doi: 10.1111/tpj. 12153

Major, I. T., Guo, Q., Zhai, J., Kapali, G., Kramer, D. M., and Howe, G. A. (2020). A phytochrome B-independent pathway restricts growth at high levels of jasmonate defense. Plant Physiol. 183, 733-749. doi: 10.1104/pp.19.01335

McNellis, T. W., Von Arnim, A. G., Araki, T., Komeda, Y., Miséra, S., and Deng, X.-W. (1994). Genetic and molecular analysis of an allelic series of cop1 mutants suggests functional roles for the multiple protein domains. Plant Cell 6, 487-500. doi: 10.1105/tpc.6.4.487

Mitreiter, S., and Gigolashvili, T. (2021). Regulation of glucosinolate biosynthesis. J. Exp. Bot. 72, 70-91. doi: 10.1093/jxb/eraa479

Navarro, L., Bari, R., Achard, P., Lisón, P., Nemri, A., Harberd, N. P., et al. (2008). DELLAs control plant immune responses by modulating the balance of jasmonic acid and salicylic acid signaling. Curr. Biol. 18, 650-655. doi: 10.1016/j.cub.2008.03.060

Ortigosa, A., Fonseca, S., Franco-Zorrilla, J. M., Fernández-Calvo, P., Zander, M., Lewsey, M. G., et al. (2020). The JA-pathway MYC transcription factors regulate photomorphogenic responses by targeting HY5 gene expression. Plant J. 102, 138-152. doi: 10.1111/tpj.14618

Paul-Victor, C., Züst, T., Rees, M., Kliebenstein, D. J., and Turnbull, L. A. (2010). A new method for measuring relative growth rate can uncover the costs of defensive compounds in Arabidopsis thaliana. New Phytol. 187, 1102-1111. doi: 10.1111/j.1469-8137.2010.03325.x

Paz-Ares, J., Valencia, A., Costantino, P., Vittorioso, P., Davies, B., Gilmartin, P. et al. (2002). REGIA, an EU project on functional genomics of transcription factors from Arabidopsis thaliana. Comp. Funct. Genomics 3, 102-108. doi: $10.1002 /$ cfg. 146

Piasecka, A., Jedrzejczak-Rey, N., and Bednarek, P. (2015). Secondary metabolites in plant innate immunity: conserved function of divergent chemicals. New Phytol. 206, 948-964. doi: 10.1111/nph.13325

Schweizer, F., Fernández-Calvo, P., Zander, M., Diez-Diaz, M., Fonseca, S., Glauser, G., et al. (2013). Arabidopsis basic helix-Loop-helix transcription factors MYC2, MYC3, and MYC4 regulate glucosinolate biosynthesis, insect performance, and feeding behavior. Plant Cell 25, 3117-3132. doi: 10.1105/tpc.113.115139 
Silverstone, A. L., Jung, H.-S., Dill, A., Kawaide, H., Kamiya, Y., and Sun, T.-P. (2001). Repressing a repressor: gibberellin-induced rapid reduction of the RGA protein in Arabidopsis. Plant Cell 13, 1555-1566. doi: 10.1105/TPC.010047

Smolen, G. A., Pawlowski, L., Wilensky, S. E., and Bender, J. (2002). Dominant alleles of the basic helix-loop-helix transcription factor ATR2 activate stress-responsive genes in Arabidopsis. Genetics 161, 1235-1246. doi: 10.1093/genetics/161.3.1235

Sønderby, I. E., Burow, M., Rowe, H. C., Kliebenstein, D. J., and Halkier, B. A. (2010). A complex interplay of three R2R3 MYB transcription factors determines the profile of aliphatic glucosinolates in Arabidopsis. Plant Physiol. 153, 348-363. doi: 10.1104/pp.109.149286

Spoel, S. H., Koornneef, A., Claessens, S. M., Korzelius, J. P., Van Pelt, J. A., Mueller, M. J., et al. (2003). NPR1 modulates cross-talk between salicylateand jasmonate-dependent defense pathways through a novel function in the cytosol. Plant Cell 15, 760-770. doi: 10.1105/tpc.009159

Thies, W. (1979). Detection and utilization of a glucosinolate sulfohydrolase in the edible snail, Helix pomatia. Naturwissenschaften 66, 364-365. doi: $10.1007 / \mathrm{BF} 00368477$

Tyler, L., Thomas, S. G., Hu, J., Dill, A., Alonso, J. M., Ecker, J. R., et al. (2004). DELLA proteins and gibberellin-regulated seed germination and floral development in Arabidopsis. Plant Physiol. 135, 1008-1019. doi: $10.1104 /$ pp. 104.039578

Weller, J. L., Hecht, V., Vander Schoor, J. K., Davidson, S. E., and Ross, J. J. (2009). Light regulation of gibberellin biosynthesis in pea is mediated through the COP1/HY5 pathway. Plant Cell 21, 800-813. doi: 10.1105/tpc.108.063628

Wild, M., Davière, J.-M., Cheminant, S., Regnault, T., Baumberger, N., Heintz, D., et al. (2012). The Arabidopsis DELLA RGA-LIKE3 is a direct target of MYC2 and modulates jasmonate signaling responses. Plant Cell 24, 3307-3319. doi: $10.1105 /$ tpc.112.101428

Willige, B. C., Ghosh, S., Nill, C., Zourelidou, M., Dohmann, E. M., Maier, A., et al. (2007). The DELLA domain of GA INSENSITIVE mediates the interaction with the GA INSENSITIVE DWARF1A gibberellin receptor of Arabidopsis. Plant Cell 19, 1209-1220. doi: 10.1105/tpc.107. 051441

Yang, D.-L., Yao, J., Mei, C.-S., Tong, X.-H., Zeng, L.-J., Li, Q., et al. (2012). Plant hormone jasmonate prioritizes defense over growth by interfering with gibberellin signaling cascade. Proc. Natl. Acad. Sci. U.S.A. 109, E1192-E1200. doi: 10.1073/pnas.1201616109

Yazaki, J., Galli, M., Kim, A. Y., Nito, K., Aleman, F., Chang, K. N., et al. (2016). Mapping transcription factor interactome networks using HaloTag protein arrays. Proc. Natl. Acad. Sci. U.S.A. 113, E4238-E4247. doi: 10.1073/pnas.1603229113

Zhai, Q., Yan, L., Tan, D., Chen, R., Sun, J., Gao, L., et al. (2013). Phosphorylation-coupled proteolysis of the transcription factor MYC2 is important for jasmonate-signaled plant immunity. PLoS Gen. 9:e1003422. doi: 10.1371/journal.pgen.1003422

Zhang, X., Ji, Y., Xue, C., Ma, H., Xi, Y., Huang, P., et al. (2018). Integrated regulation of apical hook development by transcriptional coupling of EIN3/EIL1 and PIFs in Arabidopsis. Plant Cell 30, 1971-1988. doi: $10.1105 /$ tpc. 18.00018

Zheng, Y., Cui, X., Su, L., Fang, S., Chu, J., Gong, Q., et al. (2017). Jasmonate inhibits COP1 activity to suppress hypocotyl elongation and promote cotyledon opening in etiolated Arabidopsis seedlings. Plant J. 90, 1144-1155. doi: $10.1111 /$ tpj.13539

Zhu, L., Bu, Q., Xu, X., Paik, I., Huang, X., Hoecker, U., et al. (2015). CUL4 forms an E3 ligase with COP1 and SPA to promote light-induced degradation of PIF1. Nat. Commun. 6:7245. doi: 10.1038/ncomms 8245

Züst, T., Joseph, B., Shimizu, K. K., Kliebenstein, D. J., and Turnbull, L. A. (2011). Using knockout mutants to reveal the growth costs of defensive traits. Proc. $R$ Soc. B Biol. Sci. 278, 2598-2603. doi: 10.1098/rspb.2010.2475

Conflict of Interest: The authors declare that the research was conducted in the absence of any commercial or financial relationships that could be construed as a potential conflict of interest.

Copyright (C) 2021 Frerigmann, Hoecker and Gigolashvili. This is an open-access article distributed under the terms of the Creative Commons Attribution License (CC $B Y)$. The use, distribution or reproduction in other forums is permitted, provided the original author(s) and the copyright owner(s) are credited and that the original publication in this journal is cited, in accordance with accepted academic practice. No use, distribution or reproduction is permitted which does not comply with these terms. 